\title{
Chemical Reactions in Thermal Carbonization Processing of Citric Acid-Urea Mixtures
}

\author{
Weixiong Liang, ${ }^{a}$ Ping Wang, ${ }^{a}$ Liju Yang, ${ }^{*, b}$ Christopher M. Overton, ${ }^{a}$ Brian Hewitt, ${ }^{a}$ and Ya-Ping Sun ${ }^{\star, a}$ \\ ${ }^{a}$ Department of Chemistry, Clemson University, Clemson, South Carolina 29634, USA \\ ${ }^{b}$ Department of Pharmaceutical Sciences, Biomanufacturing Research Institute and Technology Enterprise, \\ North Carolina Central University, Durham, North Carolina 27707, USA
}

Email: lyang@nccu.edu (L. Y.), syaping@clemson.edu (Y.-P. S.)

\begin{abstract}
Carbon dots are small carbon nanoparticles with effective surface passivation by mostly organic species. For such a simple and well-defined nanoscale structure, the classical and most reliable synthesis is the use of pre-existing small carbon nanoparticles for surface chemical functionalization with organic molecules. However, a more popular synthetic approach in the literature has been the "one-pot" carbonization of organic precursors, which with appropriate processing conditions could in principle create local structures in the resulting dot-like entities that may be comparable to the structural configuration in carbon dots, though the carbonization can also easily produce colored organic materials crosslinked into the sample structures. An extreme example was the thermal processing of the specific organic precursor mixtures including only citric acid with formamide or urea to yield samples of red/near-IR absorption and emission features, which prompted the claims of "red/near-IR carbon dots". In reality, these spectral features have nothing to do with nanoscale carbon, let alone carbon dots, but simply associated with molecular dyes/chromophores produced in chemical reactions of the specific precursor mixtures under the thermal processing conditions intended for carbonization. In this work, the isolation and identification of the responsible molecular dyes/chromophores were pursued. The results present further evidence for the conclusion that the red/near-IR absorption and emission features have nothing to do with carbon dots.
\end{abstract}

Keywords carbon dots, nano-hybrids, carbonization, red absorptions, near-IR fluorescence

\section{Introduction}

Carbon "quantum" dots or carbon dots (CDots) $)^{[1-3]}$ have attracted much recent attention for their interesting properties and potentially broad applications. ${ }^{[3-16]}$ Phenomenologically and mechanistically, CDots exploit the intrinsic properties of small carbon nanoparticles in terms of their characteristic electronic transitions and photoexcited state processes. In fact, small carbon nanoparticles have increasingly been recognized as representing the nanoscale carbon allotrope at zero-dimension, with the other members of the family including the one-dimensional carbon nanotubes and two-dimensional graphenes (Figure 1). ${ }^{[3,17]}$ While structural and edge/surface defects are unavoidable in all these nanoscale carbon allotropes, at least experimentally, such defects in small carbon nanoparticles are more pronounced with particularly significant consequences when the nanoparticles are "naked". ${ }^{[3,18,19]}$ Because of that, however, the passivation of the defects in small carbon nanoparticles can have very dramatic effects, enabling the realization and enhancement of the photoexcited state properties and characteristic redox processes intrinsic to the nanoparticles. Such passivation of defects serves as the basis for CDots, in which the surface defects of small carbon nanoparticles are passivated effectively by chemical functionalization with selected organic molecules/species (Figure 1)..$^{[1,20,21]}$

In principle the same configuration of nanoscale carbon with surface passivation by organic moieties could also be found in a comparable or even equivalent fashion in the nano-carbon - organic moiety "mixtures" (Figure 1), ${ }^{[3,22,23]}$ in which the nanoscale carbon entities or domains could be

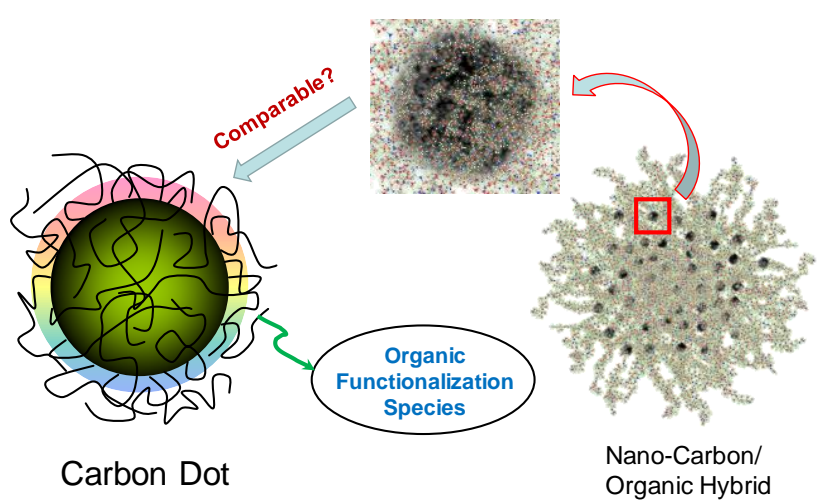

Figure 1 Cartoon illustrations on a carbon dot (left) with a solid carbon nanoparticle core surface-functionalized by organic species, and the "nano-carbon/organic hybrid" (right) from the carbonization processing of organic precursors under appropriate conditions. Also illustrated is on the possible structural comparability of the highlighted small domain in the hybrid with the carbon dot.

generated/produced in the thermal carbonization processing of the corresponding organic precursors. Such a comparability in nanoscale configuration illustrated in Figure 1 must be the assumption implicitly or explicitly behind the popular "one-pot" carbonization approach for the synthesis of samples that exhibit some of the characteristic properties of CDots, such as the bright and colorful fluorescence emissions. ${ }^{[1,22-24]}$ While 
convenient, the thermal carbonization synthesis is understandably difficult to control, generally yielding complicated mixtures at both the sample and sample structure levels. In fact, the "dot" samples from the thermal carbonization synthesis should be considered more appropriately as carbon/organic hybrids that are randomly structured at the nanoscale, dubbed "nano-hybrids" (Figure 1). ${ }^{[2]}$

The carbonization of organic precursors is not different from overcooking vegetables, which is always characterized by the varying degrees of overcooking and correspondingly the formation of various colored species in addition to the char as a product of the locally extreme overcooking. The colored species as unavoidable byproducts of the carbonization processing are organic dyes/chromophores, which depending on their covered wavelengths may interfere with the photophysical and photochemical functions of the carbonization-generated nano-hybrids referred to above (Figure 1), which are configuration-wise somewhat analogous to CDots. ${ }^{[17,25-28]}$ One extreme case for such interference is in the samples obtained from the thermal processing of only a few specific organic precursors, mixtures of citric acid with formamide or urea as precursors to be exact. ${ }^{[29-33]}$ The red/near-IR absorptions and fluorescence emissions of the samples obtained from the thermal processing of these specific precursors were mistakenly assigned to "red/near-IR carbon dots", ${ }^{[29-33]}$ yet in reality they could only be associated with organic molecular dyes/chromophores produced in chemical reactions of the precursor molecules under the thermal processing conditions. ${ }^{[28,34]}$ The investigation reported here was aimed at an understanding of the samples containing the organic molecular dyes/chromophores that are responsible for the observed red/near-IR absorptions and fluorescence emissions, and assessing the feasibility with respect to their isolation and/or identification.

\section{Results and Discussion}

In studies reported in the literature, citric acid (CA) has been a popular component in precursor mixtures for carbonization synthesis, such as mixtures of CA with small diamines and oligomeric polyethylenimine. ${ }^{[23,35-38]}$ The popularity is likely due to the three carboxylic acid moieties in the CA molecular structure amenable to ready decarboxylation under thermal processing conditions. However, only when CA is paired with formamide (FA) or urea, both containing the same unsubstituted amide groups (Figure 2), the thermal processing of the mixtures under relatively mild conditions such as $160^{\circ} \mathrm{C}$ for a few hours has yielded samples of strong visible absorptions with significant $\mathrm{red} /$ near-IR spectral features (Figure 2). ${ }^{\left[{ }^{29-34]}\right.}$ Since there is no similar absorption feature in samples from CA mixtures with fully $\mathrm{N}$-substituted FA or urea, $N, N$-dimethylformamide (DMF) or $N, N, N^{\prime}, N^{\prime}$-tetramethylurea (TetraMU), respectively (Figure 2), nor from FA or urea only without $\mathrm{CA}$, the only explanation for the observed absorption results is that the red/near-IR absorptions of the samples from the CA-FA and CA-urea precursor mixtures must be due to molecular dyes/chromophores produced in thermally induced chemical reactions under the processing conditions originally intended for carbonization. ${ }^{[34]}$

The sample from the processing of the CA-urea mixture at $160{ }^{\circ} \mathrm{C}$ for $6 \mathrm{~h}$ was selected for the effort on isolating and identifying the primary molecular dyes/chromophores that are responsible for the observed solution color and red/near-IR absorptions (Figure 2). The as-produced sample was apparently rather complex, with NMR spectra showing both sharp and broad peaks (Figure 3) corresponding to small molecules and "polymeric" species, respectively. In the high
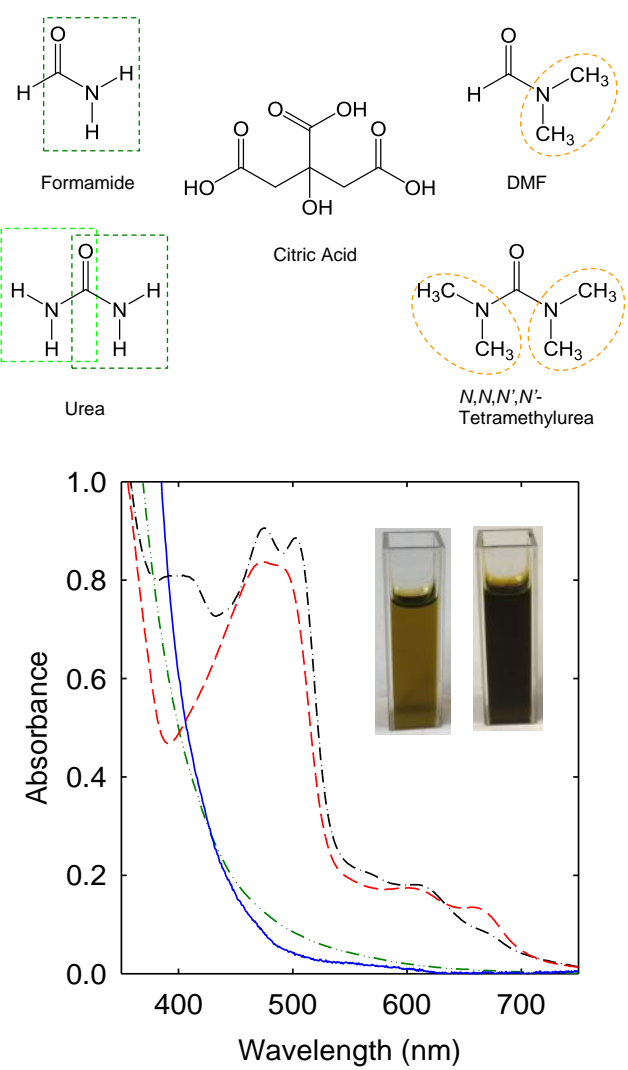

Figure 2 Upper: Molecular structures of the organic precursors. Lower: Absorption spectra of DMF solutions of samples from the thermal processing of CA-urea (dash), CA-FA (dash-dot), CA-TetraMU (solid), and CA-DMF (dash-dot-dot) mixtures. Insert: Photos of the CA-urea sample in DMF solutions of lower (left) and higher (right) concentrations.

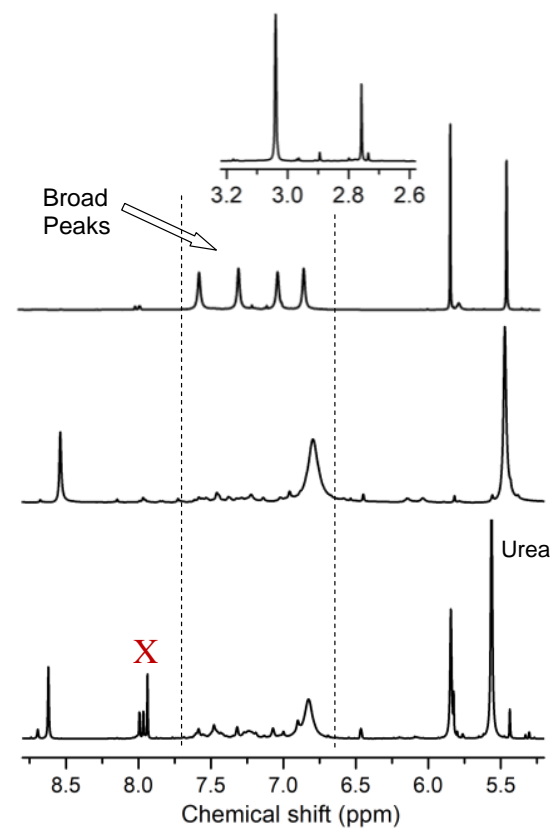

Figure 3 Proton NMR spectra in DMSO- $d_{6}$ for the as-produced CA-urea sample (lower), the cleaned sample after DCM precipitation (middle), and the insoluble sample from methanol anti-solvent precipitation (upper, with the inset for high field). "X": The DMF solvent peak. 
field $(<4 \mathrm{ppm})$ region of the proton NMR spectra, there are multiple strong sharp peaks due to the presence of abundant aliphatic moieties. The sample was cleaned and/or fractionated via dialysis and anti-solvent precipitation.

The simple cleaning of the sample was dialysis in a membrane tubing of pore size $\sim 500$ in molecular weight equivalent against fresh water for $12 \mathrm{~h}$ to remove unreacted precursor molecules and other small molecular species. The highly colored portion of the sample was found to remain inside the dialysis tubing, as confirmed by the absorption results shown in Figure 4, thus suggesting the association of the $\mathrm{red} /$ near-IR absorption features with molecular dyes/chromophores in relatively large species incapable of escaping via the pores of the dialysis tubing.

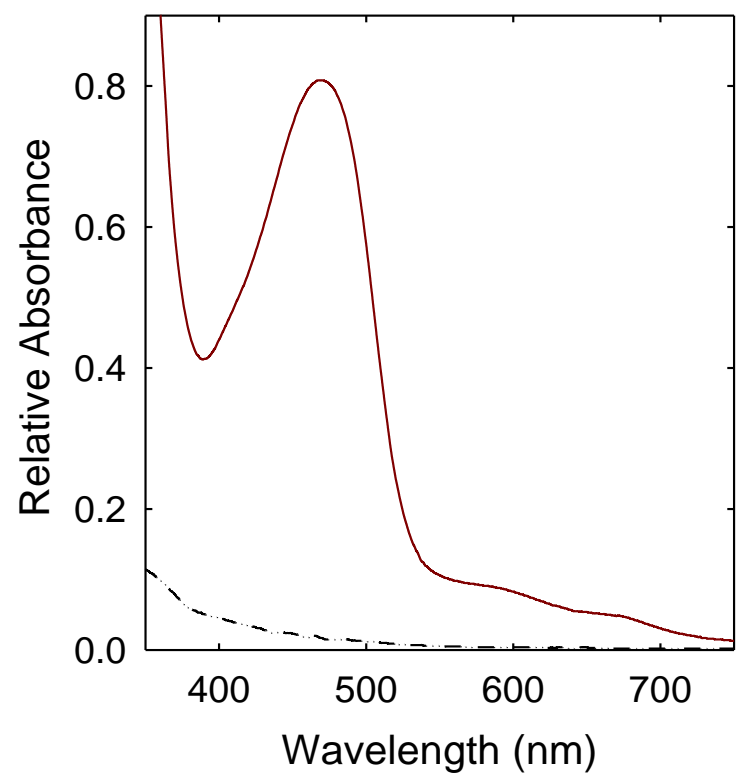

Figure 4 After the dialysis of the CA-urea sample, absorption spectra of DMF solutions of the samples recovered from inside the dialysis tubing (solid) and from the water outside the dialysis tubing (dash-dot-dot).

The further cleaning of the sample was by anti-solvent precipitation from the concentrated DMF solution of the sample into dichloromethane (DCM) as the anti-solvent. However, the visible absorptions of the recovered sample after the cleaning were essentially the same as those of the pre-precipitation sample, and the NMR spectra were somewhat similar as well (Figure 3), though most of the aliphatic species responsible for the strong aliphatic NMR peaks in the high field $(<4 \mathrm{ppm})$ were apparently removed in the DCM anti-solvent precipitation. The cleaned sample remained very complex nonetheless, as reflected by the many fractions found in the separation on a preparative thin-layer chromatography (TLC) plate shown in Figure 5, together with their corresponding absorption spectra.

The use of methanol as anti-solvent could fractionate the sample to have the precipitate contain most of the red/near-IR absorptive species (Figure 6). The weak absorptions of the recovered sample in the blue/near-UV spectral region suggest little content of carbon nanoparticles-like entities in the fraction thus obtained, because it is well known that carbon nanoparticles have relatively much stronger absorptions in the blue/near-UV spectral region (Figure 6). ${ }^{[19]}$ Thus, the results are strongly against the claims in the literature reports on "red/near-IR carbon dots" being responsible for the observed $\mathrm{red} /$ near-IR absorptions, since carbon dots are derived from carbon nanoparticles (or nanoscale carbon entities/domains

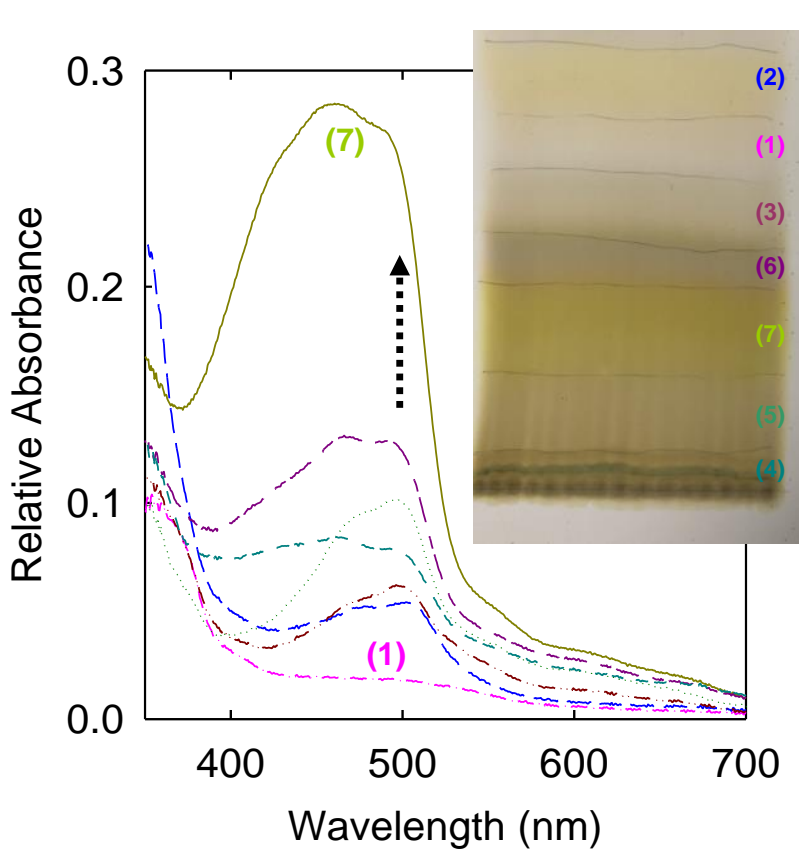

Figure 5 Absorption spectra of DMF solutions of the 7 fractions from the separation on the TLC plate (inset) for the CA-urea sample after DCM anti-solvent precipitation, with the spectra arranged such that the absorbance at $500 \mathrm{~nm}$ increasing progressively (marked by the arrow) from fraction (1) to fraction (7), corresponding to the designation of fraction numbers on the TLC plate.

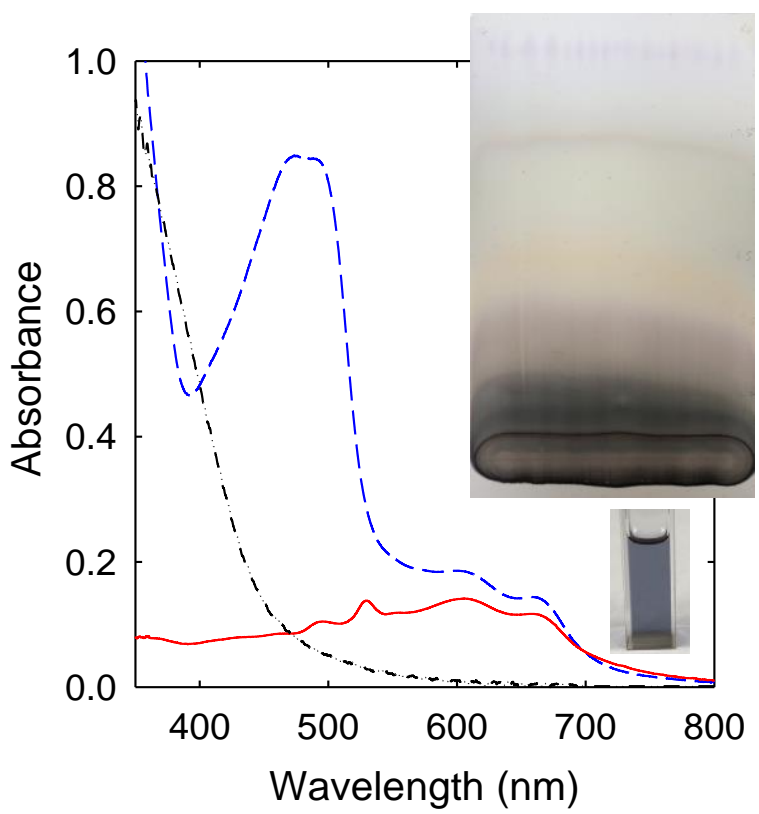

Figure 6 Absorption spectra of DMF solutions of the as-prepared CA-urea sample (dash) and the insoluble sample from the methanol anti-solvent precipitation (solid, photos in the inset on the solution and the TLC plate separation with DMF-ethyl acetate of 20 vol\% DMF as eluent), in comparison with the spectrum of the aqueous suspended small carbon nanoparticles (dash-dot-dot).

that are equivalent to the nanoparticles) both by definition and in practice experimentally.

The further separation of the red/near-IR absorptive fraction was proven difficult, taking Figure 6 for example, with most of the sample staying at the origin of the TLC plate under various 
elution solvent conditions such as 20/80 (V/V) DMF-ethyl acetate mixture. The difficulty in TLC separation may be attributed to the extreme complexity of the sample, including perhaps also the polymeric nature of the species that contain the red/near-IR absorptive chromophores. As discussed above, these species were found to be too large to escape via the pores of the dialysis tubing.

The NMR results of the methanol insoluble fraction containing the red/near-IR absorptive species are also shown in Figure 3. Since the fraction accounts for only a small percentage (somewhat less than 5\%) in the sample from DCM anti-solvent precipitation or for even smaller percentage in the as-produced sample, the dominating NMR signals in the spectrum of this fraction are clearly different from those in the other spectra (Figure 3). Especially prominent are the 4 relatively broad peaks of equal integrations in the $6.5-7.8 \mathrm{ppm}$ region, likely associated with aromatic protons in species of less mobility (more polymeric) than simple molecules containing aromatic moieties. The high field is dominated by 2 relatively sharper peaks at $2.76 \mathrm{ppm}$ and $3.04 \mathrm{ppm}$ (Figure 3 inset).

The red/near-IR fraction obtained from the methanol anti-solvent precipitation was found to be significantly fluorescent, with the observed emissions strongly dependent on excitation wavelengths, but the dependence is very different from what is known and established for CDots, ${ }^{[1,39]}$ thus again strongly against the claims of "red/near-IR carbon dots". As shown in Figure 7 on the excitation wavelength dependence, the emission spectra appear to be in two groups, one (group 1) corresponding to $400-500 \mathrm{~nm}$ excitations and the other (group 2) corresponding to $560-680 \mathrm{~nm}$ excitations, with the spectra at $520 \mathrm{~nm}$ and $540 \mathrm{~nm}$ excitations apparently as transition between the two groups (as also marked in the figure). The divide between the two groups coincides with the sharp change in the absorption spectrum of the mixture sample before the methanol anti-solvent precipitation, as marked by the arrow in Figure 7 (Upper), which seems to suggest that the methanol-insoluble fraction may also contain the chromophores responsible for the more intense absorption band over the $400-520 \mathrm{~nm}$ spectral region (Figure 7).

The observed fluorescence quantum yields are also excitation wavelength dependent (Figure 7), but again the dependence is different from the pattern known for CDots. ${ }^{[1,39,40]}$ There are similarly two groups of excitation wavelengths, one over the 400-500 nm region corresponding to the higher plateau in quantum yields, and the other over the red spectral region corresponding to the lower plateau in quantum yields (Figure 7). The pattern is roughly comparable with that for the excitation wavelength dependence of fluorescence spectra, which collectively suggests the dominance of fluorescence emissions by various mixtures of molecular dyes/chromophores in their respective spectral regions, with little to do with the minor content of nanoscale carbon entities/domains in the original sample and even less so in the fraction from the methanol anti-solvent precipitation.

Overall, the spectroscopy results of the methanol-insoluble fraction, along with the lack of success in the TLC separation effort, are consistent with the assessment that like the as-produced and cleaned (with dialysis and DCM anti-solvent precipitation) samples, the fraction from the methanol antisolvent precipitation remained a complex mixture of many components, some of which must be polymeric in nature to result in the significant broadening in NMR measurements. More specifically on the large species containing the red/ near-IR molecular chromophores, an educated guess is that they might be molecular dyes formed in the thermally induced
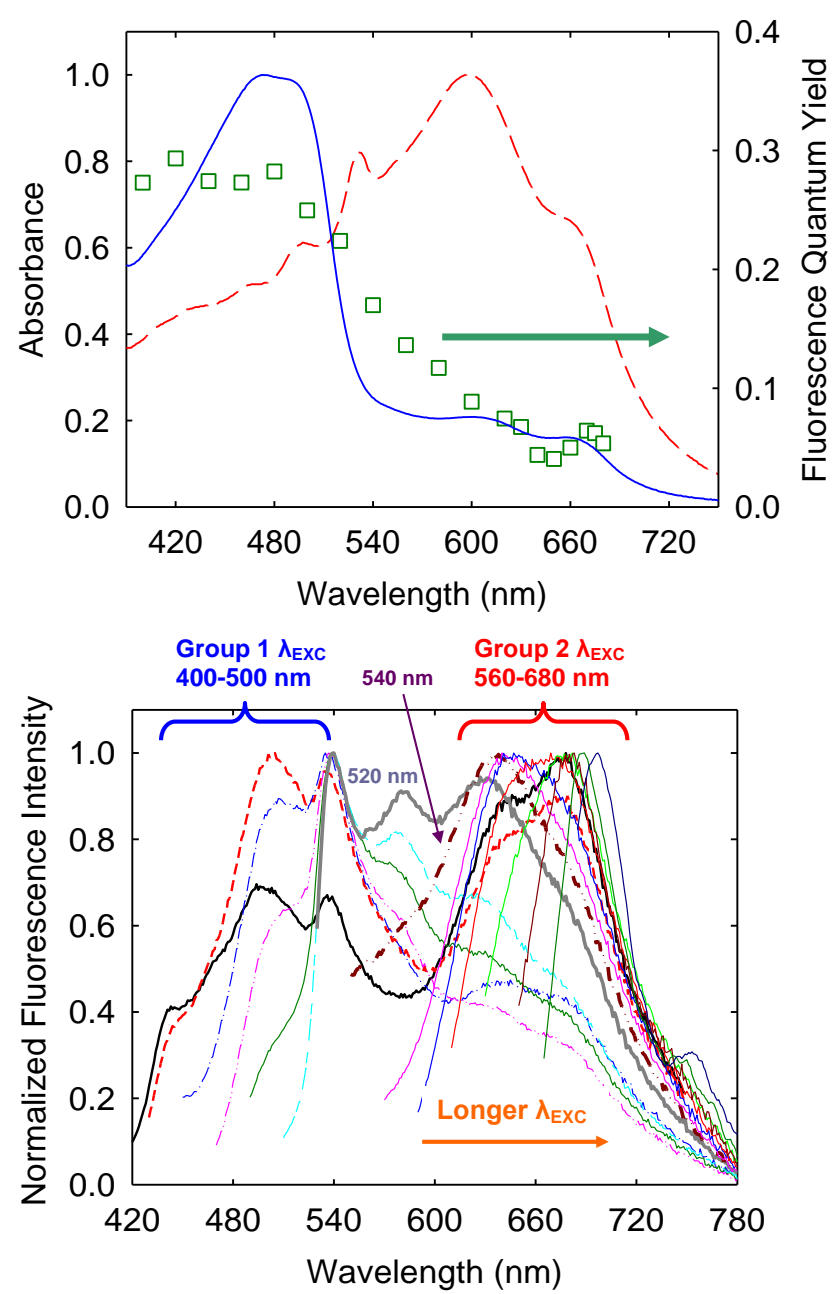

Figure 7 Upper: Absorption spectra of the as-prepared CA-urea sample (solid, the small arrow pointing to the turn between two regions) and the insoluble sample from the methanol anti-solvent precipitation (dash), and fluorescence quantum yields of the latter at different excitation wavelengths. Lower: Fluorescence spectra at different excitation wavelengths (from 400 to $680 \mathrm{~nm}$ in $20 \mathrm{~nm}$ increment) of the insoluble sample from the methanol anti-solvent precipitation. All spectra were measured in DMF solutions.

chemical reactions of citric acid with urea, which are crosslinked by some nanoscale carbon entities and/or other crosslinked organic species produced in the relatively minor carbonization and/or thermal reactions. These randomly produced entities may be structurally similar to the nano-hybrids illustrated in Figure 1, except that the organic moieties in the nano-hybrids contain red/near-IR molecular dyes/chromophores from the thermally induced chemical reactions of the few specific precursor molecules.

In conclusion, there is no "red/near-IR carbon dot", only red/near-IR molecular dyes/chromophores in rather complex mixtures with other organic species crosslinked among themselves and/or by the minor content of nanoscale carbon entities, analogous to the product mixtures from overcooking some vegetables or organic matters. It is likely that such complex mixtures with crosslinking are impossible to be separated for the isolation and unambiguous identification of the $\mathrm{red} /$ near-IR absorptive molecular dyes/chromophores. Alternatively, however, investigations on more controlled chemical reactions of the specific precursor molecules, namely 
the mixtures of citric acid with formamide or urea, may ultimately reveal the identities of the molecular dyes/chromophores, so as to put the erroneous claims of "red/near-IR carbon dots" to rest for good. On the broad implications of the conclusion here, all existing and future claims of "red/near-IR carbon dots" from any precursors and/or from any syntheses must be accompanied by unambiguous evidence that the intrinsically weak optical absorptions of small carbon nanoparticles over the red/near-IR spectral region can be and have been enhanced by increasing the corresponding electronic transition probabilities. According to the currently available knowledge and experimental results on the electronic transitions in carbon nanoparticles and other nanoscale carbon allotropes in general, the desired enhancement will prove to be impossible.

\section{Experimental}

\section{Materials}

Citric acid, formamide, and DMF were purchased from VWR, urea from Alfa Aesar, and $N, N, N^{\prime}, N^{\prime}$-tetramethylurea from $\mathrm{TCl}$. Dichloromethane and methanol were obtained from Mallinckrodt. Dialysis membrane tubing (molecular weight cutoff $\sim 500$ ) was supplied by Spectrum Laboratories. Water was deionized and purified in a Millipore Direct $Q$ Water Purification System.

\section{Measurement}

Optical absorption spectra were recorded on Shimadzu UV-2501 and UV-1280 spectrophotometers. Fluorescence spectra were acquired on a Jobin-Yvon emission spectrometer equipped with a $450 \mathrm{~W}$ xenon source, Gemini-180 excitation and Triax-550 emission monochromators, and a photon counting detector (Hamamatsu R928P PMT at $950 \mathrm{~V}$. 9,10-Bis(phenylethynyl)anthracene in hexane was used as a standard in the determination of fluorescence quantum yields by the relative method (matching the absorbance at the excitation wavelength between the sample and standard solutions and comparing their corresponding integrated total fluorescence intensities). Fluorescence spectra were corrected for nonlinear instrument response by using separately determined correction factors. NMR measurements were performed on a Bruker NEO $500 \mathrm{MHz}$ NMR spectrometer equipped with a Prodigy nitrogen-cooled cryoprobe.

\section{CA-Urea}

Citric acid (CA, $1 \mathrm{~g})$ and urea $(2 \mathrm{~g})$ were dissolved in DMF $(10 \mathrm{~mL})$ in a glass vial under mild sonication (VWR 250D ultrasonic cleaner). The resulting colorless solution was transferred to a two-neck round bottom flask $(25 \mathrm{~mL})$, with one neck fitted with a glass condenser and the other for purging with nitrogen, in an oil bath pre-heated to $160^{\circ} \mathrm{C}$. After stirring for $6 \mathrm{~h}$ under nitrogen protection, the solution was cooled back to ambient temperature for characterization and measurements.

For the dialysis, the as-produced sample solution $(3 \mathrm{~mL})$ was diluted to $10 \mathrm{~mL}$ with water before being added to the dialysis tubing (MWCO $\sim 500$ ), where the dilution was to protect the dialysis tubing from any damages. The dialysis was in a large glass beaker $(2 \mathrm{~L})$ filled with fresh water for $12 \mathrm{~h}$. Then, the solution inside the dialysis and water in the beaker were both collected and concentrated for further characterizations.

For the anti-solvent precipitation with dichloromethane (DCM) as the anti-solvent, the as-produced sample solution in DMF was concentrated to around $5 \mathrm{~mL}$, and then slowly dropped into DCM $(100 \mathrm{~mL})$ in an Erlenmeyer flask with vigorous stirring. The resulting mixture was vacuum-filtrated through a $0.22 \mu \mathrm{m}$ membrane filter, and the filter cake was collected and washed with DCM 3 times. Similarly for the anti-solvent precipitation into methanol, the as-produced sample solution in DMF was concentrated, and slowly dropped into ice cold methanol $(100 \mathrm{~mL})$ in an Erlenmeyer flask with vigorous stirring. The resulting mixture was vacuum-filtrated through a $0.22 \mu \mathrm{m}$ membrane filter, and the filter cake was collected and washed with ice cold methanol 3 times. The cleaned samples from the anti-solvent precipitation treatments were used for further characterization.

For separation/fractionation using preparative thin-layer chromatography (TLC), the sample recovered from the DCM anti-solvent precipitation was dissolved in DMF for a concentrated solution. The sample solution was carefully deposited onto a TLC plate $(10 \times 20 \mathrm{~cm})$. The separation was in the DMF-ethyl acetate mixture (30/70, V/V) as elution solvent, and multiple colored fractions on the TLC plate were observed. The fractions were scraped off the plate and dissolved separately in DMF (3 $\mathrm{mL}$ each), followed by centrifuging at $10,000 \mathrm{~g}$ to keep the supernatants as DMF solutions of the fractions for further characterization. Similarly, the methanolinsoluble sample from the methanol anti-solvent precipitation was dissolved in DMF for a concentrated solution to be carefully deposited onto the same TLC plate for the elution in DMF-ethyl acetate mixtures of different volume fractions.

\section{CA-TetraMU}

CA $(1 \mathrm{~g})$ and $N, N, N^{\prime}, N^{\prime}$-tetramethylurea (TetraMU, $\left.2 \mathrm{~g}\right)$ were dissolved in DMF $(10 \mathrm{~mL})$ in a glass vial under mild sonication (VWR 250D ultrasonic cleaner). The solution was transferred to a two-neck round bottom flask in an oil bath pre-heated to $160{ }^{\circ} \mathrm{C}$. After stirring for $6 \mathrm{~h}$ under nitrogen protection, the solution was cooled back to ambient temperature for characterization and measurements.

\section{CA-FA}

CA $(0.5 \mathrm{~g})$ was dissolved in DMF $(10 \mathrm{~mL})$ in a glass vial at $40{ }^{\circ} \mathrm{C}$ under mild sonication (VWR 250D ultrasonic cleaner), and to the solution was added formamide (FA, $5 \mathrm{~mL}$ ) with some more sonication. The resulting mixture was transferred to a two-neck round bottom flask in an oil bath pre-heated to $160^{\circ} \mathrm{C}$ stirred for $6 \mathrm{~h}$ under nitrogen protection, and then allowed to cool back to ambient temperature for characterization and measurements.

\section{CA-DMF}

CA $(1 \mathrm{~g})$ was dissolved in DMF $(10 \mathrm{~mL})$ for a solution stirred in a two-neck round bottom flask under nitrogen protection at $160{ }^{\circ} \mathrm{C}$ for $6 \mathrm{~h}$. Then, the solution was allowed to cool back to ambient temperature for characterization and measurements.

\section{Acknowledgment}

Financial support from NSF (Nos. $1701399 \& 1701424$, and 1855905) and USDA (No. 2019-67018-29689) is gratefully acknowledged. C. M. O. was a participant of Palmetto Academy, a summer undergraduate research program of the South Carolina Space Grant Consortium.

\section{Conflict of Interest}

The authors declare no conflict of interest.

Copyright (C) 2021 Weixiong Liang, Ping Wang, Liju Yang, Christopher M. Overton, Brian Hewitt, and Ya-Ping Sun. This article is an open access article distributed under the terms and conditions of the Creative Commons Attribution (CC BY) license (http://creativecommons.org/ licenses/by/4.0/). The use, distribution or reproduction in other forums is permitted, provided the original author(s) or licensor are credited and that the original publication in this journal is cited, in accordance with accepted academic practice. No use, distribution or reproduction is permitted which does not comply with these terms. 


\section{References}

[1] Sun, Y.-P.; Zhou, B.; Lin, Y.; Wang, W.; Fernando, K. A. S.; Pathak, P.; Meziani, M. J.; Harruff, B. A.; Wang, X.; Wang, H.; Luo, P. G.; Yang, H.; Kose, M. E.; Chen, B.; Veca, L. M.; Xie, S.-Y. Quantum-Sized Carbon Particles for Bright and Colorful Photoluminescence. J. Am. Chem. Soc. 2006, 128, 7756-7757.

[2] Sun, Y.-P. Fluorescent Carbon Nanoparticles. U.S. Patent 7,829,772 B2, 2010.

[3] Sun, Y.-P. Carbon Dots-Exploring Carbon at Zero-Dimension, Springer International Publishing, New York, U. S., 2020.

[4] Luo, P. G.; Sahu, S.; Yang, S.-T.; Sonkar, S. K.; Wang, J.; Wang, H.; LeCroy, G. E.; Cao, L.; Sun, Y.-P. Carbon "Quantum" Dots for Optical Bioimaging. J. Mater. Chem. B 2013, 1, 2116-2127.

[5] Ding, C.; Zhu, A.; Tian, Y. Functional Surface Engineering of C-Dots for Fluorescent Biosensing and in Vivo Bioimaging. Acc. Chem. Res. 2014, 47, 20-30.

[6] Luo, P. G.; Yang, F.; Yang, S.-T.; Sonkar, S. K.; Yang, L.; Broglie, J. J.; Liu, Y.; Sun, Y.-P. Carbon-Based Quantum Dots for Fluorescence Imaging of Cells and Tissues. RSC Adv. 2014, 4, 10791-10807.

[7] Lim, S. Y.; Shen, W.; Gao, Z. Carbon Quantum Dots and Their Applications. Chem. Soc. Rev. 2015, 44, 362-381.

[8] Fernando, K. A. S.; Sahu, S.; Liu, Y.; Lewis, W. K.; Guliants, E. A.; Jafariyan, A.; Wang, P.; Bunker, C. E.; Sun, Y.-P. Carbon Quantum Dots and Applications in Photocatalytic Energy Conversion. ACS Appl. Mater. Interfaces 2015, 7, 8363-8376.

[9] LeCroy, G. E.; Yang, S.-T.; Yang, F.; Liu, Y.; Fernando, K. A. S.; Bunker, C. E.; Hu, Y.; Luo, P. G.; Sun, Y.-P. Functionalized Carbon Nanoparticles: Syntheses and Applications in Optical Bioimaging and Energy Conversion. Coord. Chem. Rev. 2016, 320, 66-81.

[10] Peng, Z.; Han, X.; Li, S.; Al-Youbi, A. O.; Bashammakh, A. S.; El-Shahawi, M. S.; Leblanc, R. M. Carbon Dots: Biomacromolecule Interaction, Bioimaging and Nanomedicine. Coord. Chem. Rev. 2017, 343, 256-277.

[11] Hutton, G. A. M.; Martindale, B. C. M.; Reisner, E. Carbon Dots as Photosensitisers for Solar-Driven Catalysis. Chem. Soc. Rev. 2017, 46, 6111-6123.

[12] Das, R.; Bandyopadhyay, R.; Pramanik, P. Carbon Quantum Dots from Natural Resource: A Review. Mater. Today Chem. 2018, 8, 96-109.

[13] Cao, L.; Fernando, K. A. S.; Liang, W.; Seilkop, A.; Veca, L. M; Sun, Y.-P.; Bunker, C. E. Carbon Dots for Energy Conversion Applications. J. Appl. Phys. 2019, 125, 220903.

[14] Xu, D.; Lin, Q.; Chang, H.-T. Recent Advances and Sensing Applications of Carbon Dots. Small Methods 2020, 4, 1900387.

[15] Dong, X.; Liang, W.; Meziani, M. J.; Sun, Y.-P.; Yang, L. Carbon Dots as Potent Antimicrobial Agents. Theranostics 2020, 10, 671-686.

[16] Indriyati; Primadona, I.; Permatasari, F. A. A.; Irham, M. A.; Nasir, D. E. M.; Iskandar, F. Recent Advances and Rational Design Strategies of Carbon Dots towards Highly Efficient Solar Evaporation. Nanoscale 2021, 13, 7523-7532.

[17] Liang, W.; Bunker. C. E.; Sun, Y.-P. Carbon Dots: Zero-Dimensional Carbon Allotrope with Unique Photoinduced Redox Characteristics. ACS Omega 2020, 5, 965-971.

[18] Cao, L.; Meziani, M.J.; Sahu, S.; Sun, Y.-P. Photoluminescence Properties of Graphene versus Other Carbon Nanomaterials. Acc. Chem. Res. 2013, 46, 171-180.

[19] Cao, L.; Anilkumar, P.; Wang, X.; Liu, J.-H.; Sahu, S.; Meziani, M. J.; Myers, E.; Sun, Y.-P. Reverse Stern-Volmer Behavior for Luminescence Quenching in Carbon Nanoparticles. Can. J. Chem. 2011, 89, 104-109.

[20] Wang, X.; Cao, L.; Yang, S.-T.; Lu, F.; Meziani, M. J.; Tian, L.; Sun, K. W.; Bloodgood, M. A.; Sun, Y.-P. Bandgap-Like Strong
Fluorescence in Functionalized Carbon Nanoparticles. Angew. Chem. Int. Ed. 2010, 49, 5310-5314.

[21] Anilkumar, P.; Wang, X.; Cao, L.; Sahu, S.; Liu, J.-H.; Wang, P.; Korch, K.; Tackett, K. N., II; Parenzan, A.; Sun, Y.-P. Toward Quantitatively Fluorescent Carbon-Based "Quantum" Dots. Nanoscale 2011, 3, 2023-2027.

[22] Wang, P.; Meziani, M. J.; Fu, Y.; Bunker, C. E.; Hou, X.; Yang, L.; Msellek, H.; Zaharias, M.; Darby, J. P.; Sun, Y.-P. Carbon Dots versus Nano-Carbon/Organic Hybrids - Dramatically Different Behaviors in Fluorescence Sensing of Metal Cations with Structural and Mechanistic Implications. Nanoscale Adv. 2021, 3, 2316-2324.

[23] Hou, X.; Hu, Y.; Wang, P.; Yang, L.; Al Awak, M. M.; Tang, Y.; Twara, F. K.; Qian, H.; Sun, Y.-P. Modified Facile Synthesis for Quantitatively Fluorescent Carbon Dots. Carbon 2017, 122, 389-394.

[24] Ragazzon, G.; Cadranel, A.; Ushakova, E. V.; Wang, Y.; Guldi, D. M.; Rogach, A. L.; Kotov, N. A.; Prato, M. Optical Processes in Carbon Nanocolloids. Chem 2020, 7, 606-628.

[25] Xiong, Y.; Schneider, J.; Ushakova, E. V.; Rogach, A. L. Influence of Molecular Fluorophores on the Research Field of Chemically Synthesized Carbon Dots. Nano Today 2018, 23, 124-139.

[26] Khan, S.; Sharma, A.; Ghoshal, S.; Jain, S.; Hazra, M. K.; Nandi, C. K. Small Molecular Organic Nanocrystals Resemble Carbon Nanodots in Terms of Their Properties. Chem. Sci. 2018, 9, 175-180.

[27] Hinterberger, V.; Damm, C.; Haines, P.; Guldi, D. M.; Peukert, W. Purification and Structural Elucidation of Carbon Dots by Column Chromatography. Nanoscale 2019, 11, 8464-8474.

[28] Liang, W.; Ge, L.; Hou, X.; Ren, X.; Yang, L.; Bunker, C. E.; Overton, C. M.; Wang, P.; Sun, Y.-P. Evaluation of Commercial "Carbon Quantum Dots" Sample on Origins of Red Absorption and Emission Features. C - J. Carbon Res. 2019, 5, 70.

[29] Pan, L.; Sun, S.; Zhang, A.; Jiang, K.; Zhang, L.; Dong, C.; Huang, Q.; Wu, A.; Lin, H. Truly Fluorescent Excitation-Dependent Carbon Dots and Their Applications in Multicolor Cellular Imaging and Multidimensional Sensing. Adv. Mater. 2015, 27, 7782-7787.

[30] Sun, S.; Zhang, L.; Jiang, K.; Wu, A.; Lin, H. Toward High-Efficient Red Emissive Carbon Dots: Facile Preparation, Unique Properties, and Applications as Multifunctional Theranostic Agents. Chem. Mater. 2016, 28, 8659-8668

[31] Ding, H.; Wei, J.-S.; Zhong, N.; Gao, Q.-Y.; Xiong, H.-M. Highly Efficient Red-Emitting Carbon Dots with Gram-Scale Yield for Bioimaging. Langmuir 2017, 33, 12635-12642.

[32] Chen, D.; Wu, W.; Yuan, Y.; Zhou, Y.; Wan, Z.; Huang, P. Intense Multi-State Visible Absorption and Full-Color Luminescence of Nitrogen-Doped Carbon Quantum Dots for Blue-Light-Excitable Solid-State-Lighting. J. Mater. Chem. C 2016, 4, 9027-9035.

[33] Qu, S.; Zhou, D.; Li, D.; Ji, W.; Jing, P.; Han, D.; Liu, L.; Zeng, H.; Shen, D. Toward Efficient Orange Emissive Carbon Nanodots through Conjugated $\mathrm{sp}^{2}$-Domain Controlling and Surface Charges Engineering. Adv. Mater. 2016, 28, 3516-3521.

[34] Liang, W.; Wang, P.; Meziani, M. J.; Ge, L.; Yang, L.; Patel, A. K.; Morgan, S. O.; Sun, Y.-P. On the Myth of "Red/near-IR Carbon Quantum Dots" from Thermal Processing of Specific Colorless Organic Precursors. Nanoscale Adv. 2021, 3, 4186-4195.

[35] He, J.; He, Y.; Chen, Y.; Lei, B.; Zhuang, J.; Xiao, Y.; Liang, Y.; Zheng, M.; Zhang, H.; Liu, Y. Solid-State Carbon Dots with Red Fluorescence and Efficient Construction of Dual-Fluorescence Morphologies. Small 2017, 13, 1700075.

[36] Karakoçak, B. B.; Liang, J.; Kavadiya, S.; Berezin, M. Y.; Biswas, P.; Ravi, N. Optimizing the Synthesis of Red-Emissive Nitrogen-Doped Carbon Dots for Use in Bioimaging. ACS Appl. Nano Mater. 2018, 1, 3682-3692.

[37] Dong, Y.; Wang, R.; Li, H.; Shao, J.; Chi, Y.; Lin, X.; Chen, G. Polyamine-Functionalized Carbon Quantum Dots for Chemical 
Sensing. Carbon 2012, 50, 2810-2815.

[38] Zhai, X.; Zhang, P.; Liu, C.; Bai, T.; Li, W.; Dai, L.; Liu, W. Highly Luminescent Carbon Nanodots by Microwave-Assisted Pyrolysis. Chem. Commun. 2012, 48, 7955-7957.

[39] LeCroy, G. E.; Messina, F.; Sciortino, A.; Bunker, C. E.; Wang, P.; Fernando, K. A. S.; Sun, Y.-P. Characteristic Excitation Wavelength Dependence of Fluorescence Emissions in Carbon Quantum Dots. J. Phys. Chem. C 2017, 121, 28180-28186.

[40] Ge, L.; Pan, N.; Jin, J.; Wang, P.; LeCroy, G. E.; Liang, W.; Yang, L.;
Teisl, L. R.; Tang, Y.; Sun, Y.-P. Systematic Comparison of Carbon Dots from Different Preparations-Consistent Optical Properties and Photoinduced Redox Characteristics in Visible Spectrum and Structural and Mechanistic Implications. J. Phys. Chem. C 2018, 122, 21667-21676.

Received August 8, 2021 Accepted September 3, 2021 